\title{
European physicists split on new magnet
}

[MUNICH] Physicists are divided over whether Europe's next large magnet should provide a continuous field, which would offer a wide variety of uses, or a pulsed field, which would give a much more powerful field but for a relatively short duration. Or, indeed, whether new high-field magnets are needed at all.

A report from the European Science Foundation (ESF) on the scientific cases for developing both types of magnet argues that the most urgent need is for a long-pulse (10-100 millisecond duration) 100-tesla magnet facility, as US scientists are taking the lead in the development of pulsed fields.

But not all scientists are convinced that developing stronger magnets is a priority for Europe. Scientists in Germany and Britain are being polled about their opinions.

The ESF report acknowledges that the highest demand is for continuous rather than pulsed fields, but says that such facilities in Europe are already state of the art. The case for developing continuous magnetic fields should be reconsidered in a few years, it says.

But Europe is almost certain to fall behind the United States in the provision of high-field, long-pulse magnets, says the report. The world's first 100-tesla long-pulse magnet is expected to be operational at the National High Magnetic Field Laboratory in Los Alamos, New Mexico, next year.

A two-year technical study for a similar European magnet, funded by the European Commission, has just been completed by experts brought together by the ESF. They recommend building a prototype by 2003 , at an estimated cost of between ECU50 million (US\$60 million) and ECU100 million.

Research into semiconductors, lowdimensional structures, magnetism and metals, superconductors and some areas of chemistry, including optical spectrometry, would benefit from such a facility, says the report. A 100-tesla field would raise the energy of a free electron to the level existing between interacting electrons, allowing complex quantum effects to be studied.

But some European physicists believe there are too few potential users of powerful magnets to justify such costs. About $500 \mathrm{sci}$ entists use Europe's largest continuous highmagnetic-field facilities in Grenoble, France, and Nijmegen, The Netherlands, which are being upgraded to generate magnetic fields of 40-45 tesla. In contrast, between 200 and 300 use pulsed-field sources.

Proponents of investment in stronger magnets say there would be more users if new facilities were available. "If higher-field magnets are built, interesting and unanticipated science will undoubtedly flow from them," says Mike Springford, professor of physics at the University of Bristol, United Kingdom, who chaired the scientific committee.

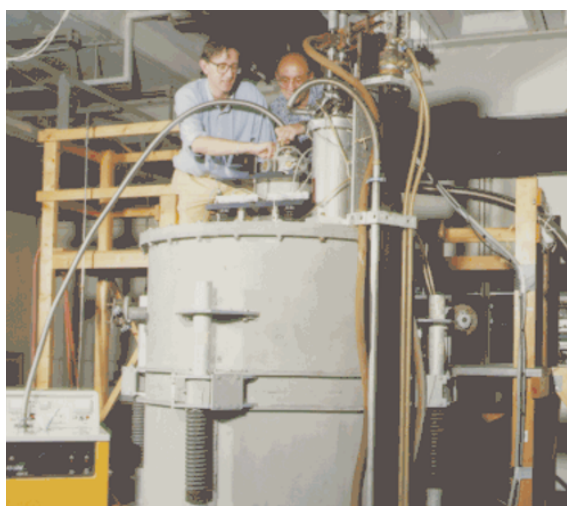

Big attraction: the 25-tesla Nijmegen I Hybrid Magnet at the University of Nijmegen in Holland.

Peter Wyder, head of the High Field Magnet Laboratory in Grenoble, points out that two recent Nobel prizes were awarded for work in semiconductor physics that depended on the use of high magnetic fields.

Klaus von Klitzing won in 1985 for discovering the Hall effect, the quantum version of the lateral deflection of moving charge carriers in a magnetic field. And Horst Störmer won this year for discovering that this effect can be seen in charge carriers apparently bearing charges a fraction of that of an electron. Both did some of their work at the Grenoble facility.

Wyder is worried that ambivalence towards developing high-field magnets could threaten the future of the Grenoble laboratory, which is financed by France's Centre National de la Recherche Scientifique

(CNRS) and the German Max Planck Society (MPS).

The MPS is uncertain whether to continue its support when the current agreement runs out in 2004, although it might be prepared to do so if the facility increased the number of international partners.

"There is no big demand for these large fields among scientists in our institutes, as far as we can see," says Gerhard Wegner, MPS vice-president for physics and director of the Max Planck Institute for Polymer Research in Mainz. "A new agreement after 2004 would require us to co-finance a major investment of around ECU100 million to modernize the facility."

The MPS is asking its researchers whether such an investment a priority. It is also seeking opinions on whether the society should support the proposed 100-tesla facility.

The Engineering and Physical Sciences Research Council is consulting UK scientists about future investment in high-field magnets. EPSRC spokesman Neil Williams expects British physicists to ask the research council to participate in either the 100-tesla project or a European high-field continuous magnet facility.

CNRS, which runs a pulsed high-field magnet facility in Toulouse as well as the Grenoble lab, wants to continue development on both fronts. "We will need partners of course," says François Gautier, professor of physics at the University of Strasbourg and deputy director of the CNRS department of physics and mathematics.

Alison Abbott

\section{German standstill as fewer chairs available}

[MUNICH] Contrary to predictions, the number of academic chairs falling vacant in Germany has dropped by nearly a half since 1993 , according to a study by the German Association of Universities.

The Wissenschaftsrat, Germany's science council, recently forecast a "high demand" for new professors because the large number hired in the 1970s would reach retirement en bloc by the end of this decade.

But the number of advertisements for professorships in biology, medicine, engineering, languages and economics has decreased dramatically. Although the natural sciences and informatics have suffered less than the average, according to the association's survey, advertised positions have still decreased by one third.

The total number of professors in Germany has slightly increased over the past six years, particularly in the eastern Länder (states). But the fall in the number of advertised posts could indicate a future decline in the overall number of chairs.
"We are facing the start of a structural change caused by general budget cutbacks," says Hartmut Schiedermair, president of the association. Saving money by not giving opportunities to the next generation is a false economy, he says. "Our young scientists are being left out in the cold."

The number of young scientists gaining the Habilitation qualification, which is required by most universities for tenured teaching jobs, has increased by 60 per cent over the past decade. In 1997, a record 1,740 young academics 'habilitated', one third in medicine and a quarter in mathematics and natural sciences. Along with the backlog of academics who habilitated earlier, they had to compete for only 1,112 vacant posts.

The Wissenschaftsrat points out that, although the number of university students is set to fall, the professor-student ratio will fall more sharply if the trend identified by the German Association of Universities continues. Students will end up being shortchanged, it warns. Tilmann Kiessling 